\title{
COMMENTS ON "COVARIANCE-INVARIANT DIGITAL FILTERING-A BETTER DIGITAL PROCESSING TECHNIQUE FOR GROUND MOTION STUDIES,” BY J. E. EHRENBERG AND E. N. HERNANDEZ
}

\author{
By JAMES L. BECK
}

Ehrenberg and Hernandez (1981) have presented a novel recursive digital algorithm for computing the relative displacement of a single degree-of-freedom linear oscillator subjected to a digitized ground motion record. Their stated aim is to achieve an accurate and numerically efficient algorithm which will allow the displacement response spectrum at several oscillator frequencies to be computed in real time by a microprocessor system. They compare their algorithm, the "covariance-invariant filter" with the "bilinear $z$ filter" proposed by Stagner and Hart (1970), and show that the new algorithm gives better accuracy for a given ratio $f_{s} / f_{n}$, where $f_{s}$ and $f_{n}$ are the sample and oscillator frequencies, respectively, and that the improvement in relative accuracy becomes greater as $f_{s} / f_{n}$ is decreased. Alternatively, for a given accuracy and oscillator frequency, the ground motion can be sampled at a lower rate for their algorithm, thereby ensuring it is more efficient computationally than the bilinear $z$ filter.

The purpose of this letter is to draw attention to an important problem relating to the accurate determination of the peak response which was not addressed by Ehrenberg and Hernandez in their paper. This problem arises because accurate computation of the oscillator displacement at each discrete time $n \Delta t, n=1,2,3$, ..., does not guarantee accurate determination of the true (continuous-time) peak displacement, since this peak will usually occur within one of the time intervals of length $\Delta t$. Nigam and Jennings (1969) consider this point when discussing their algorithm, which is currently the standard for computing response spectra from United States strong-motion records [Trifunac and Lee (1973)]. They give estimates of the largest error that can occur based on the assumption that the response in the neighborhood of the peak value is sinusoidal at a frequency equal to $f_{n}$, which is a good approximation for lightly damped oscillators. They do not give a general expression but it is easily shown that it is

$$
\frac{\text { largest error }}{\text { true peak response }}=1-\cos \left(\pi f_{n} / f_{s}\right)
$$

where $f_{s}=1 / \Delta t$. For example, to ensure that the extremum of the discrete displacements, which are assumed to be accurately computed, is within 5 per cent of the true peak displacement, it is necessary to have $f_{s} / f_{n} \geqq 10$. Alternatively, for the values $f_{s} / f_{n}=3$ and 5 given in Figures 2, 3, and 4 of Ehrenberg and Hernandez's paper, the peak displacement could be underestimated by as much as 50 and 19 per cent, respectively, for some ground motion records. To utilize the accurate displacements of the covariance-invariant filter at low $f_{s} / f_{n}$ during the calculation of response spectra, it would be necessary to develop an auxiliary algorithm which gives the peak continuous-time displacement in terms of the discrete values with an accuracy comparable to the accuracy to which these discrete displacements are calculated. 


\section{REFERENCES}

Ehrenberg, J. E. and E. N. Hernandez (1981). Covariance-invariant digital filtering-A better digital processing technique for ground motion studies, Bull. Seism. Soc. Am. 71, 1361-1367.

Nigam, N. C. and P. C. Jennings (1969). Calculation of response spectra from strong-motion earthquake records, Bull. Seism. Soc. Am. 59, 909-922.

Stagner, J. R. and G. C. Hart (1970). Application of the bilinear $z$ transform method to ground motion studies, Bull. Seism. Soc. Am. 60, 809-817.

Trifunac, M. D. and V. Lee (1973). Routine computer processing of strong-motion acelerograms, EERL Report No. 73-03, California Institute of Technology, Pasadena, California.

Division of Engineering and Applied Science

California Institute of Technology

Pasadena, California 91125

Manuscript received 2 February 1982 